\title{
Dielectronic-recombination cross sections of hydrogenlike argon
}

\author{
D. R. DeWitt, D. Schneider, M. W. Clark, and M. H. Chen \\ Lawrence Livermore National Laboratory, University of California, Livermore, California 94550 \\ D. Church \\ Texas A\&M University, College Station, Texas 77843
}

(Received 29 July 1991)

\begin{abstract}
Relative dielectronic-recombination cross sections for hydrogenlike argon are presented. The contributions of the $K L L, K L M, K L N, K L O$, and $K L P$ groups of resonances are compared to theoretical calculations. The experimental method consists of the formation and interaction of ions with electrons in an ion trap followed by an analysis of the extracted ions to determine relative yields. Comparison with theory shows that the total cross sections agree within $\pm 6 \%$.
\end{abstract}

PACS number(s): $34.80 . \mathrm{Kw}, 34.80 . \mathrm{Dp}$, 52.20.Fs

\section{INTRODUCTION}

The importance of the dielectronic-recombination (DR) process as a major contributor to the ion balance in high-temperature plasmas has been known for many years [1]. However, measurements of DR cross sections have only been achieved within the past decade. The first measurements were made by Tanis et al. [2] of the analogous resonant transfer and excitation (RTE) process. This was followed by experiments on DR using merged beams of electrons and ions [3-5]. Recent RTE measurements have extended to heliumlike uranium [6]. Direct measurement of DR cross sections of highly charged ions have only been performed within the past few years [7-9].

In this experiment we examine dielectronic recombination of the hydrogenlike $\mathrm{Ar}^{17+}$ ion. When a free electron collides with this ion it may transfer energy to the bound electron and be captured into an excited state. The total energy of the resulting doubly excited ion is above its ionization energy and may subsequently autoionize. However, if the system decays below its ionization energy through photon emission the dielectronic-recombination process is complete. These reactions have the form

$\operatorname{Ar}^{17+}(1 s)+e^{-} \rightarrow \operatorname{Ar}^{16+}\left(n l n^{\prime \prime} l^{\prime \prime}\right) \rightarrow \operatorname{Ar}^{16+}\left(1 s n^{\prime} l^{\prime}\right)+h v$.

This process is resonant in the energy of the free electron, $\varepsilon$ :

$$
\varepsilon=E\left(n l n^{\prime \prime} l^{\prime \prime}\right)-E(1 s),
$$

where $E\left(n l n^{\prime \prime} l^{\prime \prime}\right)$ is the binding energy of the twoelectron orbital $n l n^{\prime \prime} l$ ".

Dielectronic-recombination cross sections on fewelectron ions have been measured using the Lawrence Livermore National Laboratory (LLNL) electron-beam ion trap (EBIT) through the analysis of $\mathrm{x}$ rays emitted as the doubly excited system decays to the ground state [10]. In those studies, the cross sections for heliumlike nickel were obtained by normalizing the $\mathrm{x}$-ray yield to the cross sections for radiative recombination. This was necessary because the number of ions in the trap and the current density of the electron beam were unknown. Ali et al.
[11] have measured the DR cross sections for heliumlike argon using the electron-beam ion source (EBIS) at Kansas State University. In that study the current density was eliminated from the analysis by allowing the ion charge balance to reach equilibrium at the probe energy. They then eject the ions and obtain the relative chargestate balance through magnetic analysis of the resulting beam. Normalization to the nonresonant lithiumlike argon-electron impact-ionization cross section was used in order to obtain an absolute cross section.

\section{EXPERIMENT}

In the present study we use a method of ion extraction and detection similar to that of Ali et al. [11]. Highly charged ions can be extracted from the LLNL EBIT through the use of an ion extraction system that has recently been developed [12]. Detection and counting of these ions following charge-state separation allows a detailed inventory of the relative charge-state fractions within the trap. Through the analysis of these relative ion yields various electron-ion collision processes may be studied.

The operation of EBIT as a tool for $x$-ray studies of electron-ion interactions has been reported in detail $[13,14]$. In this paper we report the measurement of these processes using EBIT in a mode similar to the operation of an EBIS. EBIT is made up of a threesegment axial drift tube assembly. The voltage of these segments may be individually biased, high on the ends and low in the middle, to create an electrostatic well along the central axis. An electron beam, compressed to $\sim 70 \mu \mathrm{m}$ in a 3-T magnetic field, travels along the central axis. The energy of the electrons is determined by an overall bias voltage applied to the drift tube assembly by a fast switching high-voltage supply. Beam currents used range from 5 to $160 \mathrm{~mA}$. Neutral gas molecules such as $\mathrm{O}_{2}$ and $\mathrm{N}_{2}$ or argon, which are ionized by the beam within the central well of the drift tubes, will remain trapped radially by its space charge. These trapped ions then undergo further ionization and other electron-ion 
interactions.

In order to study dielectronic recombination in highly charged ions, the drift tubes are raised to a voltage high enough to form a sufficient population of the desired charge state. For argon, a beam energy of $7 \mathrm{keV}$ is used to form the initial charge-state balance, which takes approximately $1 \mathrm{sec}$ using a $15-\mathrm{mA}$ beam current. A typical well depth is $20 \mathrm{~V}$. Once the ionization balance is formed the interaction condition desired may be created by switching the drift tube voltage and possibly the beam current to the appropriate values. In this way the electron beam may be sequentially tuned through a range of energies containing DR resonances, for example. After a given time interval the beam is turned off to avoid further interactions as the drift tubes are brought to the extraction voltage. The extraction voltage is matched to the optics of the extraction system, which is tuned only at the start of the experiment in order to avoid changes in extraction efficiency. Once this voltage is reached the ions are dumped from the trap by ramping the voltage of the center drift tube segment above that of the top segment. The ions are then magnetically analyzed and directed into a photomultiplier for counting.

For our studies of DR in hydrogenlike argon, two experimental parameters are varied: the beam energy and the probe time. After the ionization period is completed the drift tubes are switched to a voltage between 2 and 4 $\mathrm{kV}$ as the beam energy scans the resonances in this range. Two cycles (ionization, probe, dump) are repeated at each voltage in the range scanned. The step size is $5 \mathrm{eV}$. Figure 1 shows a scan of hydrogenlike argon for a probe time of $300 \mathrm{msec}$. This scan was obtained by ionization of argon gas, which is continuously fed into the trap, at 7 $\mathrm{keV}$ and a beam current of 15-mA. The other experimental parameter is the probe time. In order to measure cross sections we work with rate equations that describe the time development of the various charge states. The

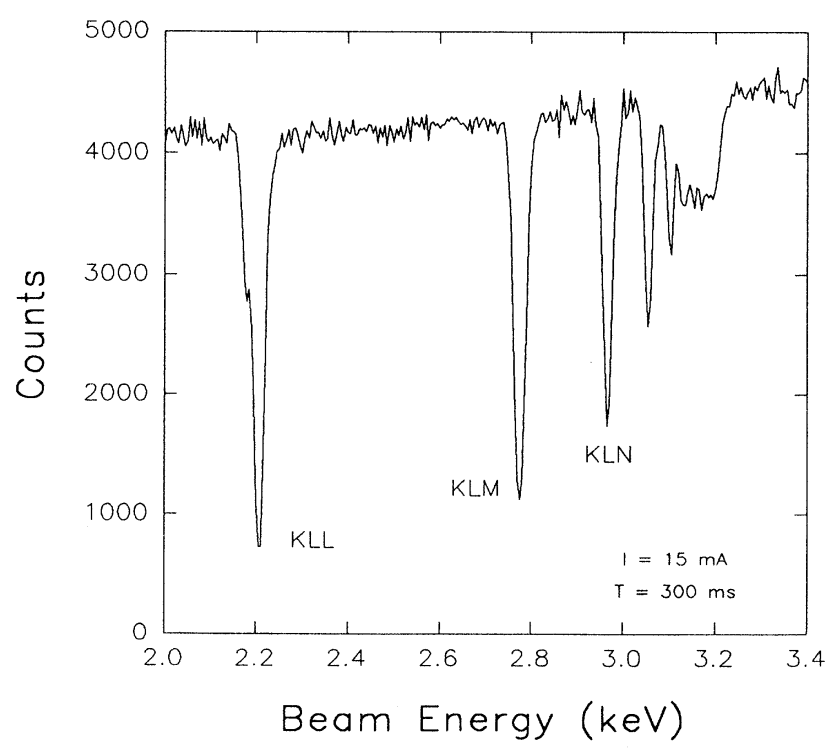

FIG. 1. Yield of hydrogenlike argon ions as a function of energy. At each energy the beam is applied for $300 \mathrm{msec}$. rate of change of any charge state can be obtained from a knowledge of its abundance in the trap following interaction for various times. The interaction times used in this experiment will not bring the plasma to equilibrium, however. Since there is no significant feeding of the hydrogenlike state it will continuously decay, with equilibrium being reached only when this state is completely depleted by DR. Since the scanning range is below the $4100-\mathrm{eV}$ ionization energy of the heliumlike argon there is no feeding of this charge state by the heliumlike ions. There is a small amount of feeding from the bare argon, but this is small because it is the result of relatively weak processes including radiative recombination and charge exchange with neutral gas molecules. These processes are nonresonant. The number of bare argon ions is also an order of magnitude smaller than the hydrogenlike ions. Therefore the scan of hydrogenlike argon shows a constant overall reduction due to the various nonresonant processes that is punctuated by sharp losses at the DR resonances.

\section{RESULTS AND DISCUSSION}

The rate equation for the $\mathrm{Ar}^{17+}$ charge state is

$$
\frac{d n}{d t}=-\frac{J^{\prime}}{e}\left\{\left[\sigma^{\mathrm{DR}}(\varepsilon)+\sigma^{\mathrm{RR}}(\varepsilon)\right] n-\sigma_{18}^{\mathrm{RR}}(\varepsilon) n_{18}\right\}-\frac{n}{\tau},
$$

where $n$ is the number of $\mathrm{Ar}^{17+}$ ions, $n_{18}$ is the number of $\mathrm{Ar}^{18+}$ (bare) ions, $J^{\prime}$ is the effective electron-beam current density, $\sigma^{\mathrm{RR}}(\varepsilon)$ is the radiative-recombination $(\mathrm{RR})$ cross section, $\sigma^{\mathrm{DR}}(\varepsilon)$ is the dielectronicrecombination cross section for the $\mathrm{Ar}^{17+}$ ion, and the $\tau^{-1}$ term includes charge exchange, losses due to leakage from the trap, and other nonresonant losses. Since $n_{18}$ is small and the RR cross section and other nonresonant losses are also quite small, we incorporate this term into the background. Solving for the DR cross section we have

$\sigma^{\mathrm{DR}}(\varepsilon)=-\frac{e}{J^{\prime}} \frac{d}{d t} \ln n(\varepsilon)-\left(\sigma^{\mathrm{RR}}(\varepsilon)-\sigma_{18}^{\mathrm{RR}}(\varepsilon) \frac{n_{18}}{n}+\frac{e}{J^{\prime} \tau}\right)$.

The term in large parentheses represents a slowly varying background that contains no resonances. Figure 2 shows $(d / d t) \ln n(\varepsilon)$ calculated from the experimental data collected for scans with different probe times of 50, 100, 200, and $300 \mathrm{msec}$. Notice the slowly changing background level of approximately $-1 / \mathrm{sec}$. Since the ion count is approximately 4000 , this represents an ion loss rate of $\sim 4$ ions/msec contributed by nonresonant processes.

In order to compare our results with theory we convolute the theoretical resonance strengths with a Gaussian distribution of electron energies. Our method is to estimate the value of the energy resolution, $w$, using a leastsquares fit to the experimental data. The experimental energy width is the result of an intrinsic distribution of electron energies resulting from the beam's space charge and a much larger noise component. In order to facilitate this fit one free parameter is needed to normalize the intensities. The obvious choice for this is $J^{\prime}$, the effective current density. In EBIT, the absolute current density is 


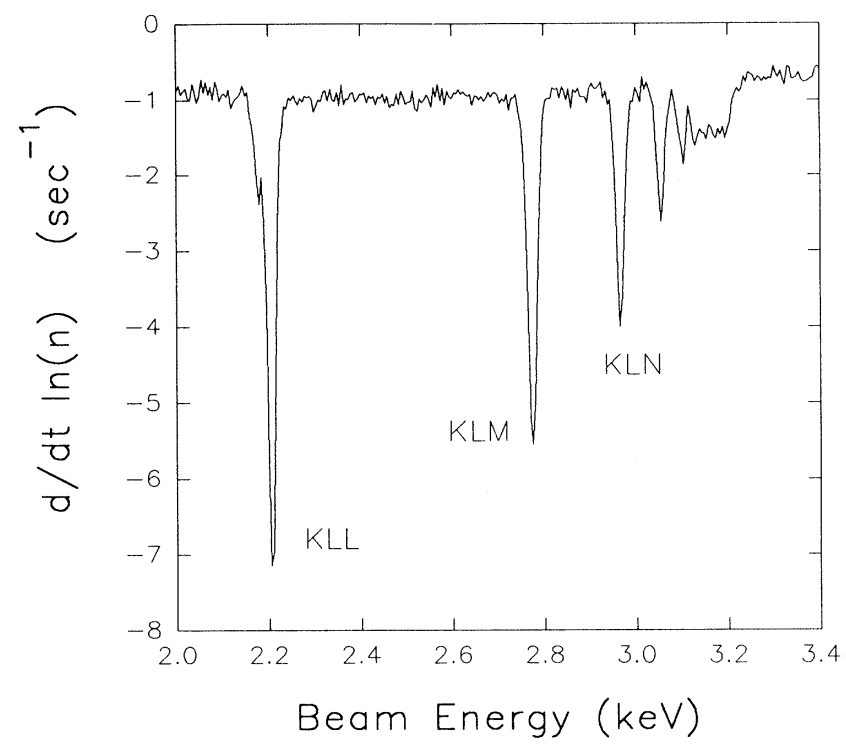

FIG. 2. Logarithmic derivative of the $\mathrm{Ar}^{17+}$ ion yield. This is directly proportional to the total DR cross section.

estimated using an electron-beam radius $\simeq 35 \mu \mathrm{m}$. For a uniform distribution of electrons in a $15-\mathrm{mA}$ beam the current density would be $\approx 400 \mathrm{~A} / \mathrm{cm}^{2}$. However, the ions in EBIT follow orbital paths about the electron beam that take them out of the interaction region for a significant fraction of the probe time. This beam overlap fraction depends on $J(r)$ and on the ion temperature. The effective current density is therefore unknown in this experiment and is taken as our fit parameter.

The theoretical dielectronic cross sections were calculated in the isolated resonance approximation. The DR resonance strength, which is the integral of the DR cross section over the natural width of the resonance, can be written as [15]

$S_{i}=\frac{4.95 \times 10^{-30}}{\varepsilon_{i}} \frac{g_{\beta}}{2 g_{\alpha}} \frac{A_{a}(\beta \rightarrow \alpha) \sum_{\gamma} A_{r}(\beta \rightarrow \gamma)}{\sum_{\alpha^{\prime}} A_{a}\left(\beta \rightarrow \alpha^{\prime}\right)+\sum_{\gamma^{\prime}} A_{r}\left(\beta \rightarrow \gamma^{\prime}\right)}$

where $g_{\beta}$ and $g_{\alpha}$ are the statistical weight factors for the intermediate doubly excited state and ground state, respectively; $A_{a}(\beta \rightarrow \alpha)$ and $A_{r}(\beta \rightarrow \gamma)$ are the Auger and radiative rates in $\sec ^{-1} ; \varepsilon_{i}$ is the resonance energy in $\mathrm{eV}$; and the resonance strength $S_{i}$ is measured in $\mathrm{cm}^{2} \mathrm{eV}$.

The atomic energy levels and wave functions were calculated using the multiconfiguration Dirac-Fock (MCDF) model in the extended average-level scheme [16]. The calculations were carried out in intermediate coupling with configuration interaction from the same complex. The detailed relativistic Auger and radiative transition rates for each autoionizing state were evaluated in first-order perturbation theory using the MCDF model [17]. In this work we included the contributions from intermediate $2 \ln ^{\prime} l^{\prime}\left(2 \leq n^{\prime} \leq 14, l^{\prime} \leq 3\right)$ states. The contributions from high $l$ states $\left(l^{\prime} \geq 4\right)$ were estimated to be less than $1 \%$.
After the background is subtracted, the data are multiplied by $\left(e / J^{\prime}\right)$ and fitted to a Gaussian function with an experimental energy resolution $w$,

$$
\sigma_{t}^{\mathrm{DR}}(\varepsilon)=\sum_{i} \frac{S_{i}}{\sqrt{2 \pi} w} \exp \left[-\frac{\left(\varepsilon-\varepsilon_{i}\right)^{2}}{2 w^{2}}\right] .
$$

Here $\sigma_{t}^{\mathrm{DR}}(\varepsilon)$ is the total contribution of all the DR resonances at the experimental point $\varepsilon$. The result of the fit is shown in Fig. 3, which shows the total theoretical DR cross sections convoluted with a Gaussian beam spread. The experimental data are normalized by $-e / J^{\prime}$. The agreement is good if $J^{\prime}$ is set to $59 \mathrm{~A} / \mathrm{cm}^{2}$ and $w=8.0$ $\mathrm{eV}$. The error bars display the experimental cross sections $\pm 6 \%$, which represents the estimated combined error in the values of $J^{\prime}(<2 \%)$ and $w(<4 \%)$, as well as the statistical error of approximately 4-5\%. We can clearly resolve the $K L L, K L M, K L N, K L O$, and $K L P$ groups of resonances and the difference in intensities is within the estimated errors.

Since we use $J^{\prime}$ as a free parameter, the experiment does not measure the total DR cross section absolutely. However, we do measure the relative contributions of the $K L X$ groups of resonances, including those at the series limit.

\section{CONCLUSION}

We have measured the relative dielectronicrecombination cross sections for the $K L L-K L P$ groups of resonances in hydrogenlike argon. The resolution is approximately $19 \mathrm{eV}$ full width at half maximum (FWHM). Comparison to theory gives good agreement for the relative contributions with differences $\leq 6 \%$.

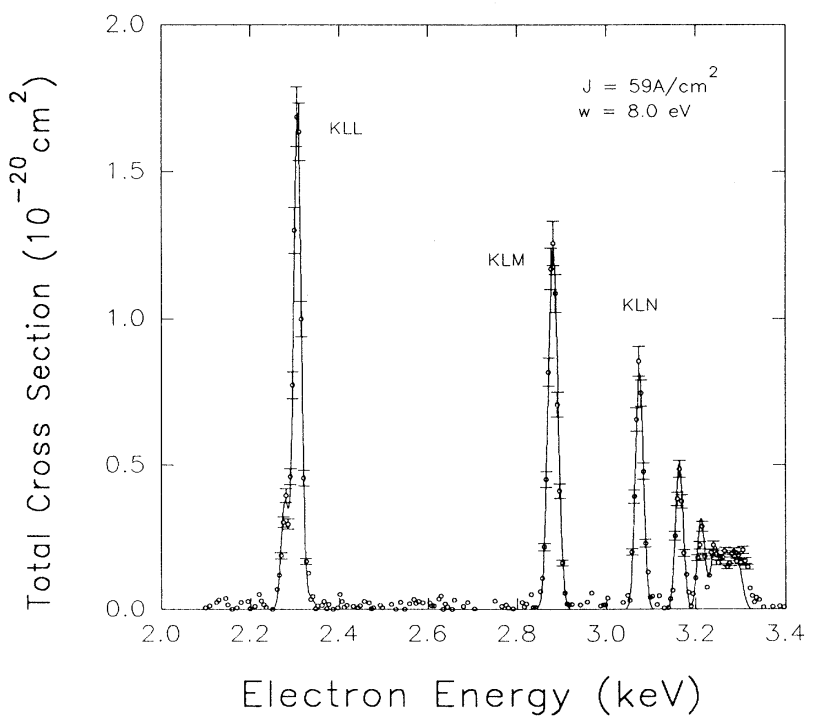

FIG. 3. Comparison of the experimental data to theoretical $\mathrm{DR}$ cross sections for $\mathrm{Ar}^{17+}$. The theory is convoluted with a beam width of 19-eV FWHM. The experimental data are divided by $J^{\prime}=59 \mathrm{~A} / \mathrm{cm}^{2}$. The error bars are $\pm 6 \%$. 


\section{ACKNOWLEDGMENTS}

We thank E. Magee and D. Nelson for their technical support. D.R.D. acknowledges C. L. Cocke and R. Ali for many helpful discussions. We also thank J. Tanis for his valuable advice. This work was performed under the auspices of the U.S. Department of Energy by the Lawrence Livermore National Laboratory under Contract No. W-7405-ENG-48.
[1] A. Burgess, Astrophys. J. 139, 776 (1964).

[2] J. A. Tanis, E. M. Bernstein, W. G. Graham, M. Clark, S. M. Shafroth, B. M. Johnson, K. W. Jones, and M. Meron, Phys. Rev. Lett. 49, 1325 (1982).

[3] D. S. Belic, G. H. Dunn, T. J. Morgan, D. W. Mueller, and C. Timmer, Phys. Rev. Lett. 50, 339 (1983).

[4] P. F. Dittner, S. Datz, P. D. Miller, C. D. Moak, P. H. Stelson, C. Bottcher, W. B. Dress, G. D. Alton, N. Neskovic, and C. M. Fou, Phys. Rev. Lett. 51, 31 (1983).

[5] J. B. A. Mitchell, C. T. Ng, J. L. Forand, D. P. Levac, R. E. Mitchell, A. Sen, D. B. Miko, and J. Wm. McGowan, Phys. Rev. Lett. 50, 335 (1983).

[6] W. G. Graham, K. H. Berkner, E. M. Bernstein, M. W. Clark, B. Feinberg, M. A. McMahan, T. J. Morgan, W. Rathbun, A. S. Schlachter, and J. A. Tanis, Phys. Rev. Lett. 65, 2773 (1990).

[7] J. P. Briand, P. Charles, J. Arianer, H. Laurent, C. Goldstein, J. Dubau, M. Loulergue, and F. Bely-Dubau, Phys. Rev. Lett. 52, 617 (1984).

[8] L. H. Andersen, J. Bolko, and P. Kvistgaard, Phys. Rev. A 41, 1293 (1990).

[9] G. Kilgus, J. Berger, P. Blatt, M. Grieser, D. Habs, B. Hochadel, E. Jaeschke, D. Kramer, R. Neumann, G.
Neureither, W. Ott, D. Schwalm, M. Steck, R. Stokstad, E. Szmola, A. Wolf, R. Schuch, A. Muller, and M. Wagner, Phys. Rev. Lett. 64, 737 (1990).

[10] D. A. Knapp, R. E. Marrs, M. A. Levine, C. L. Bennett, M. H. Chen, J. R. Henderson, M. B. Schneider, and J. H. Scofield, Phys. Rev. Lett. 62, 2104 (1989).

[11] R. Ali, C. P. Bhalla, C. L. Cocke, and M. Stockli, Phys. Rev. Lett. 64, 633 (1990).

[12] D. Schneider, D. DeWitt, M. W. Clark, R. Schuch, C. L. Cocke, R. Schmieder, K. J. Reed, M. H. Chen, R. E. Marrs, M. Levine, and R. Fortner, Phys. Rev. A 42, 3889 (1990).

[13] M. A. Levine, R. E. Marrs, J. R. Henderson, D. A. Knapp, and M. B. Schneider, Phys. Scr. T22, 157 (1988).

[14] R. E. Marrs, M. A. Levine, D. A. Knapp, and J. R. Henderson, Phys. Rev. Lett. 60, 1715 (1988).

[15] K. LaGattuta and Y. Hahn, Phys. Rev. A 24, 2273 (1981); J. Phys. B 15, 2101 (1982).

[16] I. P. Grant, B. J. McKenzie, P. H. Norrington, D. F. Mayers, and N. C. Pyper, Comput. Phys. Commun. 21, 207 (1980).

[17] M. H. Chen, Phys. Rev. A 31, 1449 (1985); 33, 994 (1986). 